\title{
FDA deems in vitro data on mutations sufficient to expand cystic fibrosis drug label
}

The US Food and Drug Administration (FDA) took the unusual step of expanding cystic fibrosis (CF) drug Kalydeco's approved use based on laboratory data and without requiring additional clinical trials. The drug, owned by Boston-based Vertex Pharmaceuticals, was already approved for treating people with any one of ten mutations in the cystic fibrosis transmembrane conductance regulator (CFTR) gene, which affect about $8 \%$ of the CF population. With the regulator's move announced on May 17, Kalydeco's label now includes an additional 23 mutations. The approach provides "a pathway for adding additional, rare mutations of the gene, based on laboratory data," the FDA said in a press release. The expanded indication for Kalydeco (ivacaftor) will bring in for treatment another $3 \%$ of the CF population, or approximately 900 people.

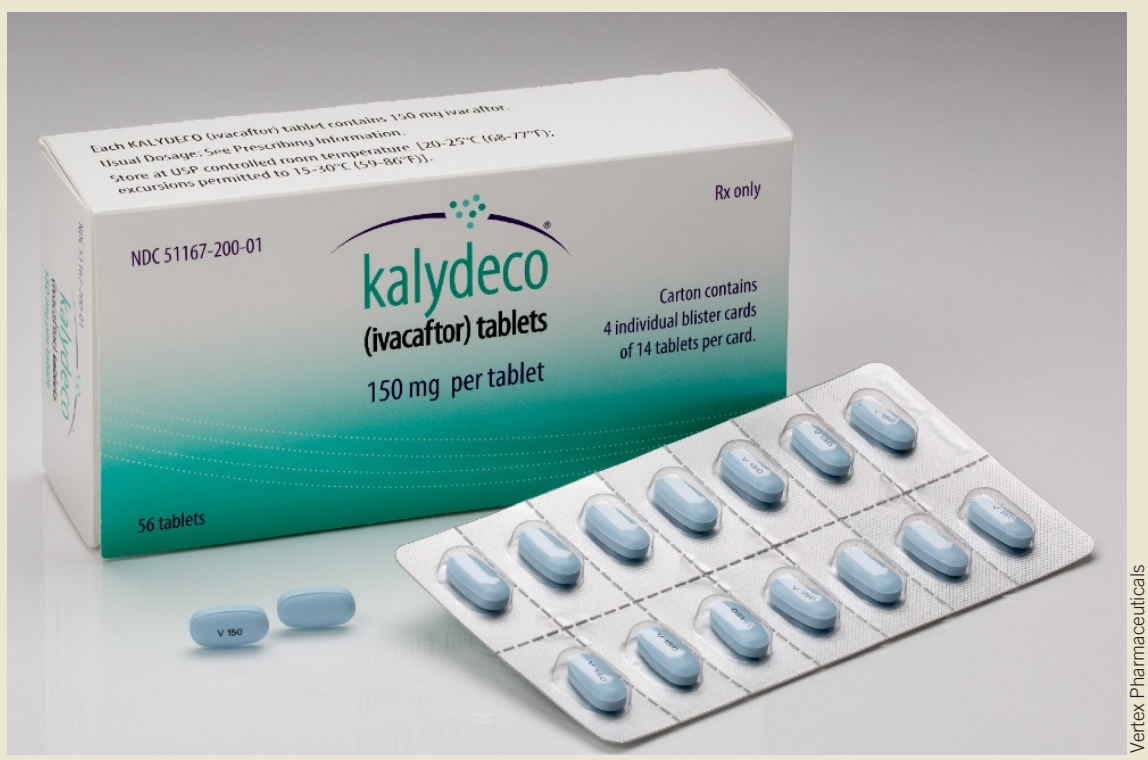

Kalydeco is now approved for another $3 \%$ of the cystic fibrosis population.
"Many rare cystic fibrosis mutations

have such small patient populations that clinical trial studies are not feasible," Janet Woodcock, director of the FDA's Center for Drug Evaluation and Research (CDER), stated in the press release. "This challenge led us to using an alternative approach based on precision medicine, which made it possible to identify certain gene mutations that are likely to respond to Kalydeco," she said.

Nearly 300 defects in CFTR, which encodes a protein that controls the movement of salt and water in and out of the body's cells, prevent the regulation of the movement of ions and water, resulting in CF. The disease is characterized by the build-up of thick, sticky secretions in the lungs, digestive tract and other parts of the body that cause severe respiratory and digestive problems as well as other complications.

Data from an in vitro cell-based model system had already reasonably predicted clinical response to Kalydeco. When cells with additional mutations in CFTR responded to Kalydeco in the laboratory test, researchers were able to extrapolate clinical benefit demonstrated in earlier clinical trials of other mutations. Cells with one or more of the 23 additional CFTR mutations have shown increases in chloride transport in response to Kalydeco by at least $10 \%$ of normal over baseline, according to Vertex.

Vertex had previously made a similar request to FDA, which the regulator rejected in February 2016. According to company spokesperson David Whitrap, the turnabout was due primarily to Vertex's efforts to help the agency better understand its preclinical process and the predictive quality of its assays. The laboratory tests also identified other CFTR mutations that fail to respond to the drug. These mutations are now also included in the new labeling.

The FDA's action is a signal to other drug makers that as long as a drug's safety profile is good, the disease is well-characterized and certain other criteria are met, for treatments that target specific mutations "in vitro assay data could potentially be used in place of additional small clinical trials when seeking to expand to other population subsets," CDER's Tony Durmowicz, lead medical officer in the Division of Pulmonary, Allergy and Rheumatology Products, Office of Drug Evaluation II, and Mike Pacanowski, associate director for genomics and targeted therapy, wrote on the FDA website.

The CFTR gene was identified almost 30 years ago, and since then researchers have accumulated a great deal of knowledge about the CFTR channel's structure and function. Plus, a large efficacy and safety database for Kalydeco, which was first approved in 2012, exists (Nat. Biotechnol. 30, 201, 2012). "Building on this existing knowledge base, we were confident that the in vitro cell model would reasonably predict the response of patients with mutations not included in the initial clinical trials," Durmowicz and Pacanowski said.

Although the approach provides a potential roadmap for drug developers, the FDA is not establishing a new type of in vitro data model, cautioned Jeremy Kahn of CDER's Division of Health Communications in an email. "Individual sponsors develop models such as this one, propose a use for them, and FDA ends up evaluating if they are reasonable for the proposed use," he said.

Mark Ratner Boca Raton, Florida 